\title{
Longitudinal Effects of Oropharyngeal Irradiation on Submucosal (internal) Lymphedema of the Head and Neck
}

\author{
Syed HS Naqvi MD¹, Carolina Gutierrez MD,2, Elliot Friedman MD², Maria Patiño MD³, Kunal Jain MD², \\ Bailey LeConte MD, Garren Low MD ${ }^{1}$, Ron J Karni MD'1
}

1Department of Otorhinolaryngology-Head and Neck Surgery, University of Texas Health Science Center at Houston, TX, USA

2Department of Physical Medicine and Rehabilitation, University of Texas Health Science Center at Houston, TX, USA

${ }^{3}$ Department of Radiology, University of Texas Health Science Center at Houston, TX, USA

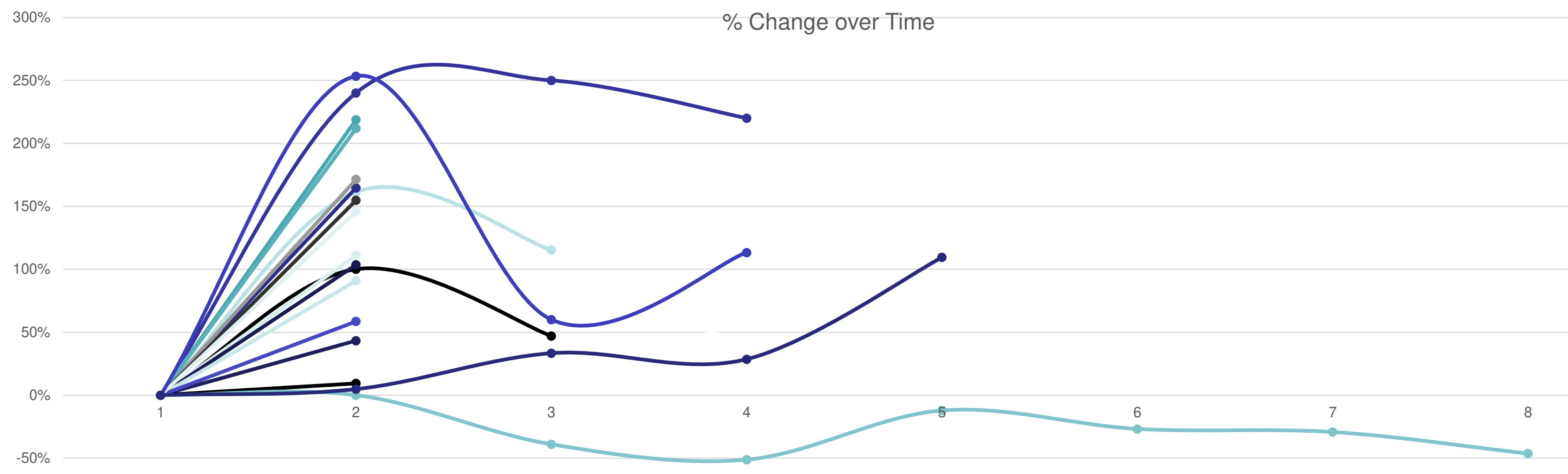

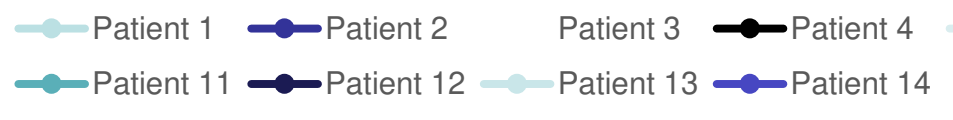

Background

Lymphedema is a well-known side effect of cancer treatment, causing a buildup of interstitial fluid in subcutaneous planes due to the loss of appropriate lymphatic flow following surgery and/or radiotherapy. This fluid accumulation, in turn, causes functional impairment such as in the arm and hand following breast cancer treatment in the axilla. In the head and neck, mucosal neoplasms and the downstream lymphatic structures are often the target of radiation and surgery, causing edema and loss of function of the larynx and pharynx. However, there are no good metrics to identify lymphedema internally at the submucosa. Instead, external neck measurements are often used to evaluate lymphedema. External neck measurements may serve as a proxy for internal lymphedema but are often confounded by weight loss, extirpative surgery, and changes in tumor volume over treatment. We sought to identify a marker for internal lymphedema that can be objectively measured in routine post-treatment surveillance radiography.

(Figure 1)

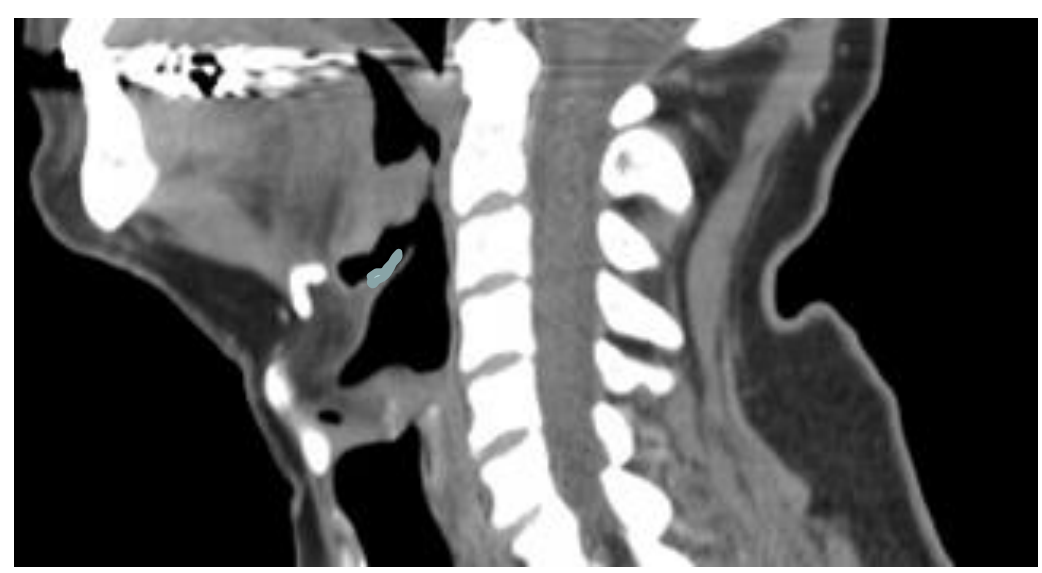

(Figure 2)

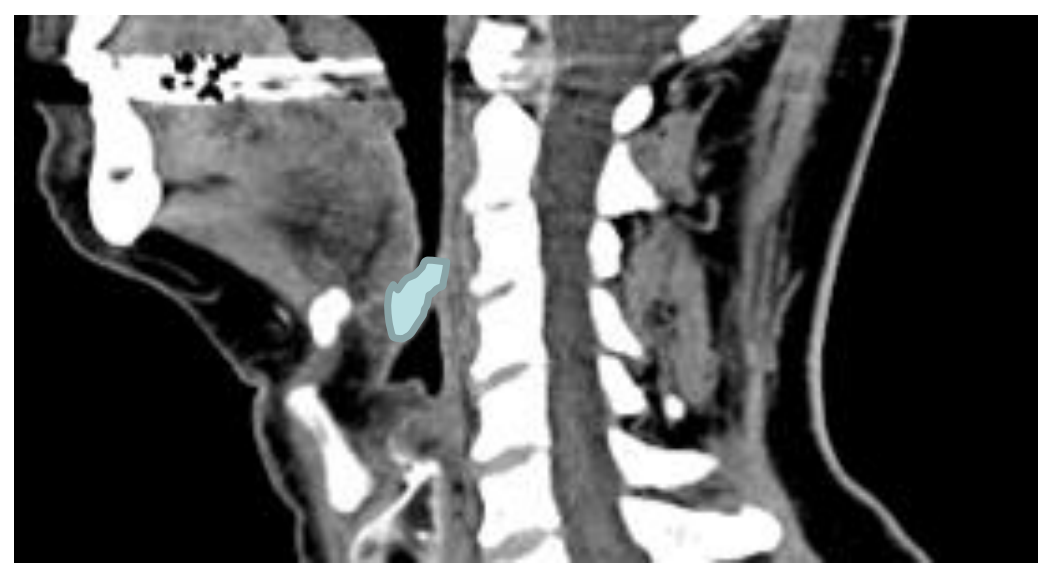

Methods and Results

In this study, we have identified 174 patients from our tumor board database who have undergone treatment for oropharynx cancer at our institution. Twenty-one patients had contrast-enhanced neck CT both before treatment (Figure 1) and at least once after treatment (Figure 2). All but one patient in this cohort had radiation therapy. Thirteen patients also underwent transoral surgery with seven of them having undergone neck dissection. Epiglottic thickness (in millimeters on axial and sagittal views) and submental soft tissue radiodensity (measured with Hounsfield Units) were measured by two neuroradiologists. In patients undergoing radiation therapy, the epiglottic thickness increased by $245 \%$ (average, axial) and $110 \%$ (average, sagittal) with a range of $106 \%$ to $418 \%$ (axial) and $43 \%$ to $253 \%$ (sagittal) (Graph 1). Radiodensity of the submental tissues was noted to increase by an average of 31 units with a range of 2.4 to 99 units change in patients undergoing radiation, reflecting increased interstitial edema. The singular patient who did not under radiation had no change in these parameters.

\section{Conclusion}

Epiglottic thickness and submental tissue radiodensity increased in all twenty patients who underwent radiotherapy in this cohort. It is our contention that submucosal edema of the pharynx and larynx as seen on post-treatment radiography may serve as a method to evaluate internal lymphedema. Future studies may serve to correlate these radiographic parameters to functional impairment of swallow, voice, airway, and neck range of motion. 